\title{
Sprachförderkonzepte in bilingualen Geographieschulbüchern
}

\author{
*m.morawski@uni-koeln.de, Institut für Geographiedidaktik, Universität zu Köln \\ **alexandra.budke@uni-koeln.de, Institut für Geographiedidaktik, Universität zu Köln
}

eingereicht am: 20.06.2017, akzeptiert am: 13.10.2017

\begin{abstract}
Aufgrund zunehmender sprachlicher Heterogenität der Schüler/innen wird ein sprachbewusster Geographieunterricht immer notwendiger. Der bilinguale Geographieunterricht könnte dafür Förderstrategien bereithalten. Im Rahmen einer Untersuchung aktueller bilingualer Erdkundeschulbücher soll der Frage nachgegangen werden, welche sprachlichen Teilkompetenzen bei den Schüler/innen wie gefördert werden.
\end{abstract}

Keywords: Sprachbewusster Geographieunterricht, bilingualer Geographieunterricht, Sprachförderung, Schulbuch

\section{Concepts of language support in bilingual geography (CLIL) school books}

Due to increasing linguistic heterogeneity in geography classrooms, language-aware geography education becomes crucial. An analysis of recently published bilingual geography school books shall assess the question, which language competences are actually supported and how.

Keywords: Language-aware geography education, bilingual geography, language support, school books

\section{Sprachliche und kommunikative Heraus- forderungen im Geographieunterricht}

Der Geographieunterricht (nicht nur in Deutschland) sieht sich verschiedenen Herausforderungen gegenüber, die einen sprachbewussten Unterricht nötig machen. Zum einen führt die aktuelle Flüchtlingsbewegung zu einer vertieften didaktischen Fokussierung auf die Folgen von Migration für den Geographieunterricht. Es werden vermehrt Kinder unterrichtet, die Deutsch nur als Zweitsprache lernen. Zahlen, die vom Bundesamt für Migration und Flüchtlinge herausgegeben wurden, geben an, dass zum Zeitpunkt der Erhebung mindestens 300.000 schulpflichtige und neu zugewanderte Kinder das Schulsystem ergänzen werden (BAMF 2015). Die erhöhte sprachliche Heterogenität in vielen Klassen macht eine sprachliche Reflexion und angepasste sprachliche Förderung im Rahmen der geographischen Unterrichtsgegenstände notwendig, um Schüler/innen mit sprachlichem Unterstützungsbedarf in ihrer Schullaufbahn nicht institutionell zu diskriminieren. Zum anderen ist Deutschland verpflichtet, die UN-Konvention über die Rechte von Menschen mit Behinderungen (United Nations
2006) umzusetzen und Schüler/innen mit sonderpädagogischen Förderbedarfen in die Regelschulen zu inkludieren. Wenn Inklusion gelingen soll, muss der Geographieunterricht sprachlich an diese Zielgruppe angepasst werden (vgl. Reich 2014: 340).

Da die sprachliche Kommunikation sowohl bei der Vermittlung fachlicher Inhalte als auch bei deren individueller Ver-, Um- und Aufarbeitung so wesentlich ist, wurden diesbezügliche Anforderungen ebenfalls in viele Curricula und in die nationalen Bildungsstandards übernommen. Diese werden hier im Kompetenzbereich ,Kommunikation' zusammenfasst (vgl. DGfG 2014: 21). Beispiele für derartige Anforderungen in den Kernlehrplänen in Nordrhein-Westfalen sind hier u.a. im Bereich der Methodenkompetenz zu nennen, etwa die mündliche und schriftliche allgemein- und fachsprachlich angemessene und strukturierte Darstellung wie Formulierung von raumbezogenen Sachverhalten und die Anwendung von Fachbegriffen (vgl. MSW NRW 2007, 2011a, 2011b). Man hat demnach erkannt, dass fachliches Wissen nur durch Kommunikation individuell ,in Wert' gesetzt werden kann. Auch die Entschlüsselung von gesellschaftlichen Diskursen 
und deren Beurteilung ist notwendig, um an der Kommunikation in einer demokratischen Gesellschaft teilnehmen zu können.

Was bedeutet es also, Geographie sprachbewusst zu unterrichten? Es bedeutet, einen Unterricht durchzuführen, der sich den sprachlichen Anforderungen bewusst wird, die von den Schüler/innen bewältigt werden müssen, um geographische Fragestellungen zu beantworten, und der diese Anforderungen flexibel vor dem Hintergrund der Lerngruppe sprachlich reflektiert (vgl. Budke \& Weiss 2014: 127). Es stellt sich die Frage, ob im bilingualen Geographieunterricht Potenziale liegen, die dabei helfen können, sprachbewussten Unterricht zu entwickeln.

Der bilinguale Geographieunterricht kann sich auf eine jahrzehntelange Erfahrung bei der Verknüpfung von sprachlichem und fachinhaltlichem Lernen stützen. Die empirische Forschung im bilingualen Geographieunterricht im deutschsprachigen Raum zeigt Tendenzen, dass sich die Fremdsprachenkompetenz relevant verbessert (vgl. DESI 2008; Kniffka \& Neuer 2008) ohne dass sich die Vermittlung fachlicher Kompetenzen drastisch reduziert (vgl. Golay 2005; Passon 2007; Meyer 2003). Übertragen wir diese Gedanken auf die sprachlich immer heterogener werdende Situation in aktuellen Klassenkonstellationen, in denen die Schüler/innen gleichzeitig bildungssprachliche, fachsprachliche und fachinhaltliche Kompetenzen erweitern, erscheint es sinnvoll, den bilingualen Unterricht und seine Potenziale bezüglich sprachlicher Förderstrategien zu analysieren. Ergebnisse aus den Interviews mit bilingual unterrichtenden Lehrkräften (vgl. Morawski \& Budke 2017a) zeigen, dass diese die vielfältigen Konzepte in den bilingualen Lehrwerken als hilfreich erachten und als umfangreicher ansehen im Vergleich zu den monolingualen Werken. Dieser Artikel soll im Rahmen einer Analyse bilingualer Geographieschulbücher aus Deutschland Antworten auf folgende Fragen geben:

1. Welche Sprachförderkonzepte finden sich in bilingualen Geographielehrwerken?

2. Wie werden diese in den Schulbüchern umgesetzt?

3. Welche Defizite und Potenziale im Bereich der sprachlichen Förderung sind in den bilingualen Schulbüchern zu erkennen und wie lassen sich die Stärken nutzen, um die Sprachförderung in monolingualen Büchern zu verbessern?

Der Artikel fokussiert somit drei Themenfelder a) sprachsensibles Arbeiten im Geographieunterricht, b) Sprachförderung im bilingualen Geographieunterricht, c) die Analyse von Sprachförderkonzepten in aktuellen bilingualen Geographieschulbüchern.

\section{Theoretischer Hintergrund}

\subsection{Die Bedeutung von Sprache im Schulunterricht}

Sprache und Sprechen kann als eine soziale Praxis verstanden werden, über die gesellschaftliche Anerkennung artikuliert und vermittelt werden kann. Fehlende Sprachkompetenzen oder die Nichtbeherrschung einer anerkannten Variante, eines Registers oder der Durchführungsprinzipien von Sprache haben negative Konsequenzen für den Bildungs- und Berufserfolg (Schendzielorz 2011: 41; Becker-Mrotzek et al. 2013: 7).

Bourdieu analysierte, wie sich die Schaffung und Durchsetzung einer einheitlichen Sprache für eine Sprachgemeinschaft entwickelt. Er erklärt dabei, wie die Produktion „einer normierten Hochsprache, der offiziellen Sprache mittels restriktiver im Dienste und als Instrument politischer Einheit" (Bourdieu \& Passeron 1971: 19; vgl. Bourdieu 2005: 60; Schendzielorz 2011: 41) über staatliche Institutionen transferiert würde. Es kann also eine Art symbolischer Kampf um Macht sein, welche Sprache primär im Unterricht akzeptiert, benotet und wertgeschätzt wird. Im Bildungssystem existiert so eine, Illusion der Chancengleichheit', bei der unter dem Deckmantel der Gleichberechtigung die legitime Sprache als Maßstab gilt. Durch die Schule und weiterführende Ausbildungsstätten wird diese reproduziert und weiter durchgesetzt. Schüler/innen, die von diesem Maßstab (eben auch der Sprache in den Schulbüchern) durch ihre Sprachkompetenz entfernt sind, werden mit Problemen konfrontiert. Eigentlich bräuchten sie mehr Zeit im Bildungswesen, um die Norm der Sprachkompetenz der legitimen Sprache zu erreichen. Dennoch werden sie allzu häufig dazu veranlasst, dieses aufgrund mangelnder Leistung und Sanktionen zu verlassen (vgl. Bourdieu \& Passeron 1971: 94; Bourdieu 2005: 60; Schendzielorz 2011: 42). Herkunftsbedingte Unterschiede im Verlauf des Bildungswegs werden dadurch verstärkt. Diese theoretische Darlegung von Sprache als symbolische Macht im Unterricht und deren Folgen sind besonders in Ländern mit starker Zuwanderung und / oder Kolonisationsgeschichte von besonderer Relevanz.

An dieser Stelle sollen die Bedeutung der Varietät, des Registers der Bildungssprache und die der kommunikativen Praxis der Schulsprache als bedeutende, symbolische und konstruierte Elemente im (deutschen) Schulraum reflektiert werden. Im allgemeingesellschaftlichen Geltungsbereich für Sprachlernende beinhaltet Bildungssprache besondere sprachliche Formate und Prozeduren, die sich auf eine auf Texthandlung basierende Sprachkompetenz beziehen (vgl. Feilke 2012: 5; Kniffka \& Roelcke 2016: 41). 


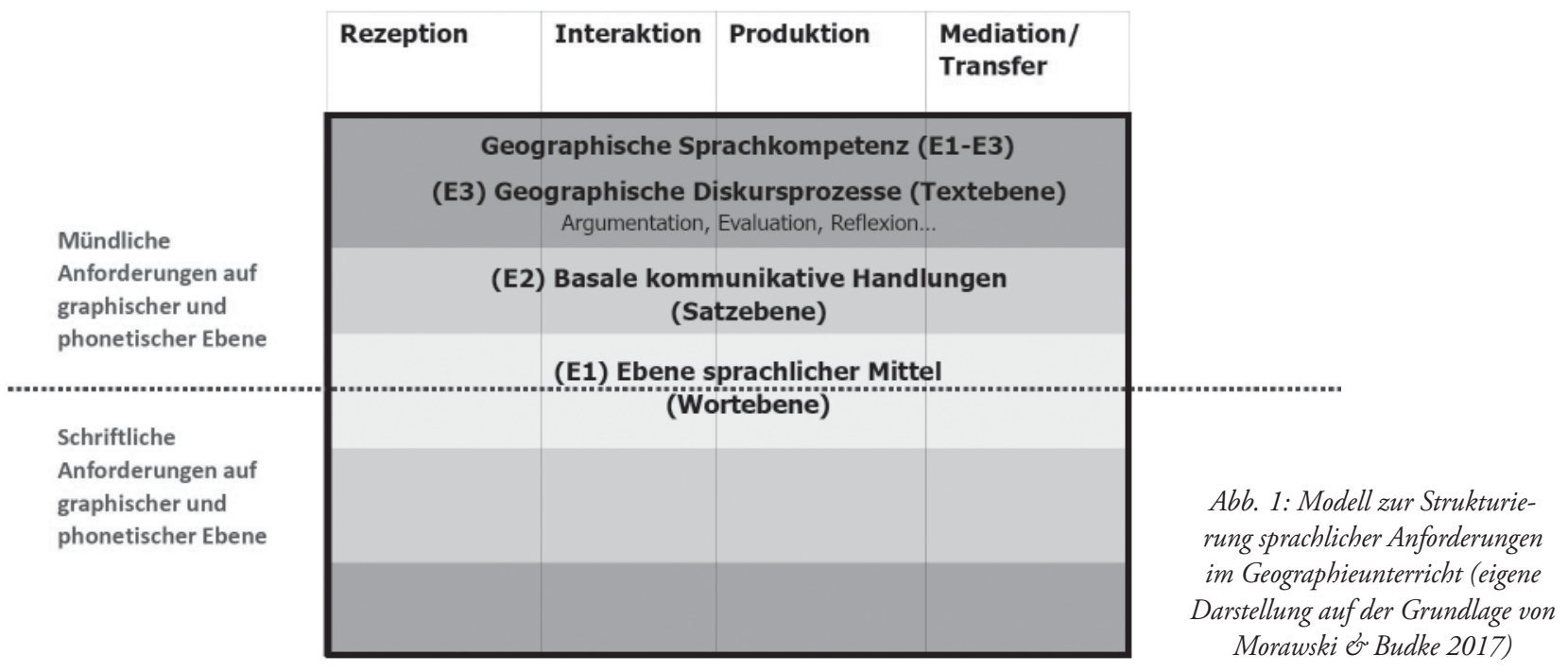

Durch Kenntnis dieser Prozeduren kann eine soziale Positionierung in Kommunikationssituationen vollzogen werden. Als Mittler zwischen Alltags- und Fachsprache bedeutet das Verständnis eines gemachten Registers von Bildungssprache, dass sowohl „fachliche als auch alltägliche Themen unabhängig von der Situation in eindeutiger Art und Weise, vollständig und in angemessener Form ausgedrückt werden" (Tajmel 2012: 9). Dazu sind ein entsprechender Wortschatz (Eindeutigkeit, Situationsunabhängigkeit) und passende grammatische Strukturen (angemessene Form) notwendig (vgl. Feilke 2013: 10; Tajmel 2012: 9; Habermas 1981: 345; Morek \& Heller 2012: 73).

Die didaktisch konstruierte kommunikative Praxis der Schul- oder Unterrichtssprache, umfasst die Gesamtheit der sprachlichen Instrumente des Lehrens sowie sprachliche Verhaltungserwartungen, die im schulischen Geltungsbereich zur Bildungssprache hinführen sollen (vgl. Feilke 2013: 10; SchmölzerEibinger 2013: 9; Kniffka \& Roelcke 2016: 50). Diese Erwartungen beeinflussen den Unterricht und selektieren die Schüler/innen nach Leistungen. Eine Reflexion dieser Anforderungen ist unter gegebenen Umständen notwendig.

\subsection{Sprache im Geographieunterricht}

Grundlegend können die Funktionen der Sprache im (Geographie-) Unterricht nach Michalak et al. (2015) als Lernmedium, als Lern- und Reflexionsgegenstand, als Kommunikationsmittel mit- und untereinander sowie als Mittel zur Leistungsüberprüfung zusammengefasst werden. Ferner wurden sprachliche Anforderungen in geographischen Kernlehrplänen von Budke und Weiss (2014) untersucht. Sie identifizierten acht übergeordnete, sprachliche Anforderungen im Geographieunterricht: (1) Infor- mationsaufnahme aus Geomedien-Beschreibung, (2) Analyse und Bewertung, (3) Erwerb und Anwendung des Fachwortschatzes, (4) Durchführung geographischer Befragungen, (5) Entschlüsselung gesellschaftlicher Diskurse, (6) Entwicklung und Durchführung von Diskussionen und Argumentationen, (7) Präsentationen von Ergebnissen und die (8) vielfältige kritische Reflexion bezogen auf Material wie Quellen hinsichtlich der Problemstellung sowie bezüglich eigener Sichtweisen und Herangehensweisen. In einer Studie von Salinas et al. (2008) hat sich gezeigt, wie effektiv die Arbeit mit visuell unterstützten Aufgaben im Geographieunterricht ist. Auch wird gezeigt, dass bezüglich des Spracherwerbs und dessen Förderung, die Arbeit mit diskontinuierlichen Darstellungsformen eine eigene Form von geographischer Sprachbildung ist (vgl. Wiegand 2006: 50; Cruz \& Thornton 2013: 91).

Ähnlich wie in allen anderen Unterrichtsfächern wird im Geographieunterricht eben auf Elemente des Registers der Bildungssprache zurückgegriffen (siehe Kap. 2.1). Es stellt sich daher die Frage, welche fachspezifischen sprachlichen Anforderungen der Geographieunterricht an die Schüler/innen stellt, die über die bildungssprachlichen hinausgehen. Um diese darzustellen, wurde ein Modell erstellt (vgl. Morawski \& Budke 2017b: 64), was hier vereinfacht wiedergegeben werden soll (siehe Abb. 1). Das Modell wurde mit zwei Absichten entwickelt: Zum einen, um Sprache im Geographieunterricht im Kontext anderer sprachlicher Kompetenzen oder Register wie der Fachsprachenkompetenz nach Roelcke (2010), dem BICS $^{1}$ und CALPS Konzept nach Cummins (1991),

Cummins wollte durch sein Konzept BICS (basic interpersonal communicative skills) und CALPS (cognitive academic language proficiency) Lehrer/innen, die besonders mit neu zugewanderten Schüler/innen arbeiten, auf besondere sprachliche Herausforderungen in der Schule aufmerksam machen. 
Bildungssprache oder Schulsprache (vgl. u.a. Feilke 2012) im Schulbereich zu positionieren, und zum anderen, um sprachliche Anforderungen und darauf bezogene sprachliche Hilfen mithilfe des europäischen Referenzrahmens zur Sprachvermittlung (CEFR 2011) zu strukturieren.

Das Modell (siehe Abb. 1) strukturiert sprachliche Anforderungen auf der Wort-, Satz- und Textebene. Auf der Wortebene sind das Verständnis und die richtige Nutzung geographischer Fachbegriffe angesiedelt (E1). Auf der zweiten Ebene können von den Schüler/innen basale kommunikative Sprachhandlungen auf Satzebene verstanden und produziert werden (E2). Wenn Schüler/ innen typische Erklärungs- und Argumentationsmuster beherrschen und geographische Texte verstehen und produzieren können, haben sie geographische Diskursfähigkeit erlangt: die dritte Ebene (E3). Unter ,Text verstehen wir alle komplexen und inhaltlich zusammenhängenden Sprachhandlungen. Typisch für den Geographieunterricht ist, dass hier auf eine Vielzahl diskontinuierlicher Darstellungsformen wie Karten, Diagramme oder Bilder Bezug genommen wird. Alle im Modell abgebildeten Bestandteile geographischer Sprachkompetenz kann man nach dem europäischen Referenzrahmen für Sprachen im Mündlichen sowie im Schriftlichen beobachten (vgl. CEFR 2011: 24). Zudem kann man die Dimensionen Rezeption, Interaktion, Produktion und Mediation als sprachliche Dimensionen unterscheiden. Mit rezeptiven Fähigkeiten ist beispielsweise die Dekodierung von u. a. sprachlichen Kartenelementen in Verbindung mit textlicher Information gemeint. Interaktive Fähigkeiten beinhalten zum Beispiel die adressatengerechte Formulierung im Kontext einer kooperativen Gruppenarbeit, während produktive Fähigkeiten die eigenständige Versprachlichung von mündlichen und schriftlichen Texten umfasst. Transferierende Fähigkeiten im Rahmen von Mediation beinhalten zum Beispiel die Umwandlung der Informationen einer Karte in einen mündlichen oder schriftlichen Text, oder die Umwandlung der Information eines Textes in ein Fließdiagramm. Diese Fähigkeiten sind natürlich nicht scharf und endgültig in ihren Handlungen zu trennen, da es häufig Überlappungen gibt. Das Modell ermöglicht die bewusste didaktische Schwerpunktsetzung sowie die sprachbewusste Reflexion, bezogen auf die jeweilige kommunikative Unterrichtssituation.

Neben der linguistischen Strukturierung kann man das Modell (Abb. 1) als Visualisierung von unterschiedlich hohen sprachlichen Anforderungen im Geographieunterricht betrachten. Dem inneren Kern des Repertoires sprachlicher Mittel (E1) wäre das simple Wahrnehmen und Nachvollziehen sprachlicher Strukturen in grammatikalischer Form zuzuordnen sowie das einfache Nennen von Fachbegriffen ohne syntaktische Einordnung. Basale Sprachhandlungen wie zusammenfassende
Sätze zur Beschreibung von Karten oder Diagrammen und Nennungen mit einzelnen Phrasierungen (E2) sind die Basis für höher geordnete Prozesse wie komplexe Argumentationen, Beurteilungen oder Bewertungen im Bereich der geographischen Diskursfähigkeit (E3). Im Bereich der sprachlichen Diskursfähigkeit (E3) geht es um die Kenntnis typischer komplexer Sprachhandlungen im Geographieunterricht. Dazu zählt beispielsweise das Wissen um die Textkohärenz eines argumentativen mündlichen oder schriftlichen Textes, strukturelle und inhaltliche Kriterien einer Erklärung oder die Beurteilung. Diese Anordnung zeigt, dass auf den Ebenen E1 und E2 Fähigkeiten formuliert sind, die für die dritte Ebene (E3) notwendig sind.

Die sprachlichen und inhaltlichen Anforderungen auf der Ebene der Diskursfähigkeit (E3) können u. a. durch Aufgaben im Anforderungsbereich (AFB) III gefördert werden, welcher in den Bildungsstandards ausgewiesen ist (DGfG 2014: 30). Die Bildungsstandards der Geographie teilen die Operatoren, mit denen Aufgaben formuliert werden, in drei Anforderungsbereiche (AFB I - AFB III) ein: 1. Reproduktion (u.a. Beschreiben), 2. Reorganisation und Transfer (u.a. Analysieren, Erklären) und 3. Reflexion und Problemlösung (u. a. Bewerten) (vgl. DGfG 2014: 32 f.). Dabei wird davon ausgegangen, dass die sprachlichen und inhaltlichen Anforderungen an die Schüler/innen mit den Anforderungsbereichen steigen. In dem AFB III ist die Argumentation von zentraler Bedeutung (vgl. Kuckuck 2014; Budke 2011, 2012; Budke \& Meyer 2015). Das Beurteilen und Reflektieren von Karten im Rahmen der Kartenauswertungskompetenz kann ebenfalls zu diesem Bereich gezählt werden (vgl. Hemmer et al. 2010a; Gryl et al. 2010). Diese sprachlich und fachlich höheren Anforderungen im Geographieunterricht können im internationalen Sprachgebrauch zu den high order thinking skills gezählt werden, die sich auf die aus Lerntheorien u.a. nach Vygotsky, Piaget und Bruner gezogenen Potenziale und Vorteile stützen (vgl. King et al. 2016: 3). Die Bedeutung von critical thinking oder problem solving für die Entwicklung der Schüler/innen, für guten Unterricht und für das kompetenzorientierte Unterrichten ist sowohl in der Interessensforschung, der Unterrichtsforschung als auch in curricularen Vorgaben verankert (vgl. DGfG 2014; Hattie 2003; Hemmer \& Hemmer 2010).

\section{Methodische Vorgehensweise: Schulbuch- analyse}

Um die Potenziale des bilingualen Geographieunterrichts bei der gleichzeitigen Förderung von fachinhaltlichen und fach- und unterrichtssprachlichen Kompetenzen bei den Schüler/innen untersuchen 
zu können, wird eine Schulbuchanalyse aktueller in Deutschland veröffentlichter und verwendeter bilingualer Geographieschulbücher durchgeführt. Schulbücher gehören zu den am häufigsten gebrauchten Unterrichtsmedien und strukturieren den Unterricht in vielfältiger Hinsicht vor (vgl. Hemmer \& Hemmer 2010: 132). Es sollen möglichst aktuelle bilinguale Schulbücher analysiert werden, um so den aktuellen Stand der Sprachförderung herauszufiltern. Aufgrund vorausgegangener Studien mit bilingualen Lehrkräften (vgl. Morawski \& Budke 2017a) sowie durch Interviews mit Verlagen und Schulvertretern konnten neun relevante Ausgaben identifiziert werden. Diese werden im deutsch-englischen bilingualen Geographieunterricht verwendet und wurden zwischen 2011 und 2015 veröffentlicht. Die ausgewählten Bücher sind nicht föderal geprägt und machen übergreifende Analysen möglich. Sie unterscheiden sich insofern, dass sie explizit entweder bestimmte Jahrgangsstufen adressieren, als Schulbuch, Workbook oder als Themenheft (Urban Environments) spezifiziert sind (siehe Tabelle 1).

\section{Tab. 1: Übersicht über analysierte bilinguale Schulbücher}

\begin{tabular}{|c|c|c|}
\hline Verlag & Titel & $\begin{array}{l}\text { Erschei- } \\
\text { nungsjahr }\end{array}$ \\
\hline Cornelsen & $\begin{array}{l}\text { Around the World, Volume } 1 \\
\text { - Schülerbuch }\end{array}$ & 2014 \\
\hline Cornelsen & $\begin{array}{l}\text { Around the World, Volume } 2 \\
\text { - Schülerbuch }\end{array}$ & 2014 \\
\hline Westermann & $\begin{array}{l}\text { Diercke Geography: Text- } \\
\text { book Basic (KI. } 5 / 6 \text { ) }\end{array}$ & 2015 \\
\hline Westermann & $\begin{array}{l}\text { Diercke Geography: Work- } \\
\text { book Basic }\end{array}$ & 2015 \\
\hline Westermann & $\begin{array}{l}\text { Diercke Geography: Text- } \\
\text { book Volume } 1 \text { (Kl. 7/8) }\end{array}$ & 2015 \\
\hline Klett & $\begin{array}{l}\text { TERRA Geography Globalisa- } \\
\text { tion and Disparities Schüler- } \\
\text { buch } 9 . \text { und } 10 \text {. Klasse }\end{array}$ & 2012 \\
\hline Klett & $\begin{array}{l}\text { TERRA Geography Global Envi- } \\
\text { ronment and Climatic Regions } \\
\text { Schülerbuch } 7 \text {. und } 8 \text {. Klasse }\end{array}$ & 2011 \\
\hline Klett & $\begin{array}{l}\text { TERRA Geography Urban Envi- } \\
\text { ronments Schülerbuch 9. und } \\
\text { 10. Klasse }\end{array}$ & 2013 \\
\hline Klett & $\begin{array}{l}\text { TERRA Geography Dynamic } \\
\text { Earth Schülerbuch 7. bis } \\
\text { 9. Klasse }\end{array}$ & 2011 \\
\hline
\end{tabular}

Zunächst werden sprachliche Hilfen identifiziert und diese werden dann in einen übergeordneten $\mathrm{Zu}$ sammenhang von Zielsetzung der sprachlichen Förderung und Komplexität der zu fördernden sprachlichen Handlung gebracht. Als sprachliche Hilfen werden in der Analyse alle angebotenen visuell-textuellen, kon- tinuierlichen und diskontinuierlichen Methoden und Materialien verstanden, die von den Autoren eingesetzt werden, um Schüler/innen bei der Bewältigung sprachlicher Anforderungen zu unterstützen.

Der Analyseansatz ist sowohl quantitativer wie auch qualitativer Art. Im Bereich der qualitativen inhaltlich-strukturierenden Inhaltsanalyse (vgl. Mayring 2015; Schreier 2012) werden Hilfen zunächst zu merkmalsimmanenten Konzepten zusammengefügt (z. B. Visuals/Layertechnik). Alle Hilfen, die durch vergleichbare Strategien dieselben Ziele verfolgen, können zu einem Verfahren zusammengeführt werden (siehe Kap. 4.1.). Zur Beantwortung der Fragen, welche Komplexität den sprachlichen Förderkonzepte bilingualer Geographieschulbücher zugrunde liegt und wie diese umgesetzt wurde, wird untersucht, welche Sprachhilfen sich auf welche Ebene sprachlicher Anforderungen beziehen (E1-E3 in Abb. 1). Zum Beispiel wird ein Block mit Schlüsselbegriffen, in dem lediglich Wörter angezeigt werden, zur Förderung des Fachwortschatzes gezählt und somit zur Wortebene (E1). Anschließend werden Sprachhilfen identifiziert, welche die Kompetenzen der Schüler/ innen auf Satzebene fördern wollen (E2). Ein abgebildeter Kasten mit zum Beispiel helpful phrases, der Satzanfänge, ganze Sätze und Phrasen mit dem Ziel der Satzkonstruktion beinhaltet, wird als Verfahren zur Förderung fachsprachlicher Kompetenzen auf Satzebene identifiziert. Anschließend werden Sprachhilfen untersucht, welche die geographischen Diskursfähigkeiten fördern sollen (E3). Ein geschlossener Modelltext wird dementsprechend zur sprachlichen Förderung auf Textebene gezählt. Gemäß dem Modell schließen Einheiten, die sich auf die Textebene (E3) beziehen, die Wort-(E1) und Satzebene (E2) mit ein. Weiter wird analysiert, ob die sprachlichen Hilfen auf den unterschiedlichen Ebenen vorrangig produktive, rezeptive, interaktive oder mediative fachsprachliche Fähigkeiten der Schüler/innen fördern sollen.

Im Rahmen einer quantitativen Inhaltsanalyse (Frequenz- und Häufigkeitsanalyse, vgl. u.a. Marienfeld 1976: 215; Hillers 1984) werden Zählungen durchgeführt. Es wird z. B. untersucht, wie häufig sprachliche Förderung auf Wort-, Satz- oder Textebene vorkommt. Zudem wird untersucht, wie viele der sprachlichen Hilfen mit expliziten Verweisen in der Aufgabenstellung im Doppelseitenkontext verbunden sind. Weiter wird untersucht, auf welche Anforderungsbereiche sich die Aufgaben und die zugehörigen sprachlichen Hilfen beziehen. Es konnten insgesamt 15 merkmalsimmanente Förderverfahren mit 1.233 zugeordneten sprachlichen Hilfen als Grundgesamtheit in Zusammenhang mit 2.767 Aufgabenstellungen gebracht werden. 


\section{Forschungsergebnisse}

\subsection{Konzepte/Verfahren der Sprachförderung in bilingualen Geographieschulbüchern}

Durch die induktive Analyse werden 15 Konzepte sprachlicher Hilfen identifiziert und verlagsübergreifend zusammengefasst:

1. Sprachnotiz: Bei der Sprachnotiz wird im Rahmen des Doppelseitenkontextes in einem Hinweiskästchen auf die richtige Verwendung geographischer Fachtermini und auf deren syntaktischen Einbau hingewiesen. Das Einfügen und die Übertragung der Begriffe finden in einem Beispiel statt, so dass eine Reproduktion und ein Transfer der Rechtschreibvorgabe durchgeführt werden können.

2. „Wie sagt man?": Ein konkreter Formulierungshinweis, z. B. die Antwort auf „Wie sagt man, wo sich ein Ort im Koordinatensystem befindet?", gibt den Schüler/innen auf Wortund Satzebene vor, wie sie eine ganz konkrete Antwort formulieren können, die sie dann übernehmen können.

3. Key Terms im Kontext der Doppelseite: Eine Liste von Worten wird in einer Box im Doppelseitenkontext präsentiert. Der fachinhaltliche Wortschatz, also geographisch relevante Fachtermini im jeweiligen Kontext, soll damit gefördert werden. Die Erweiterung des Wortschatzes findet in den Bereichen Verben, Nomen, zusammengesetzte Hauptwörter sowie im Bereich der Kollokationen statt. Im pragmatischen und semantischen Sinne wird die Bedeutung und Verwendung von Begriffen im geographischen Kontext (z. B. holiday season) erschlossen. Hinweise auf wichtige Wörter und die Erklärung von relevanten Wörtern aus dem Kontext erfolgen teilweise mit Fußnoten.

4. Ausführliche Bildunterschrift: Im Kontext der Doppelseite findet eine unterstützende Kontextualisierung von Bild und Text statt. Dies bedeutet, dass ein didaktisch reduzierter Text die Informationen, welche einer Abbildung zu entnehmen sind, zusammenfasst. Ein begleitender Informationstext als ausführliche Bildunterschrift kann dann zur variablen Übernahme von sprachlichen Elementen für eine zugehörige Aufgabe genutzt werden. Zudem sind die Informationen aber auch rückwirkend durch die direkte, parallele wie visuelle Darbietung erschließbar.

5. Visuals/Layertechnik im Kontext der Doppelseiten: Hier geht es um den Wortschatz, der für die Sprachhandlung im Bereich eines Bildes oder einer Abbildung als notwendig erachtet wird. Dieser wird in Form von Nomen, Komposita, Verben und Adjektiven als Wortfeld direkt in die jeweilige Abbildung eingeblendet. Beispielsweise wird der Text, direction of flow im Bild eines Flusses abgebildet. Demnach findet eine sofortige visuelle Kontextualisierung von sprachlichen Elementen statt.

6. Sprachliche und inhaltliche Strukturierungshilfen als Teil der Aufgabenstellung: Dieses Konzept bedeutet, dass die Schüler/innen im Rahmen der Aufgabenstellungen konkrete Hilfestellungen erhalten, wie die Ergebnisse strukturiert werden sollen. Zum Beispiel können, graphic organizers' angeboten werden. Diese bringen antizipierte Ergebnisse in einen visuell-strukturierenden Zusammenhang. Dies kann durch Pfeile und Textboxen passieren, welche ausgefüllt werden sollen und bereits unausgefüllt einen Zusammenhang vorgeben.

7. Helpful Words and Phrases: Im Kontext einer thematischen Doppelseite und als Exkurs in den Methodikseiten werden in Wortboxen für den jeweiligen Zusammenhang von den Autor/innen als sinnvoll erachtete Phrasen, Satzanfänge und Satzkonstruktionen vorgeschlagen. Diese Vorschläge können eine rein sprachliche Zielsetzung haben (Ausdrücken der eigenen Meinung), einen inhaltlichen Schwerpunkt verfolgen (Beschreibung von Prozessen im Kontext der Plattentektonik) oder als Mischform formuliert sein. Syntaktische Förderung findet durch die Vorgabe und Stützung von Satzstrukturen für den schriftlichen und mündlichen Gebrauch in Form von Haupt- und Relativsätzen statt. Textstrukturelle oder pragmatische Förderung findet in dem Sinne statt, dass beispielsweise die textkohärente Struktur von Beschreibungen oder Konnektoren für diese Struktur vorgeschlagen werden können. Die Anforderungen variieren und es findet ein direkter situativer Bezug der Sprachübung zu einem konkreten inhaltlichen Beispiel statt.

8. Methodikexkurse I - Helpful Phrases: Losgelöst vom Kontext der Doppelseiten werden Wortboxen (vgl. Punkt 7) mit methodischem Schwerpunkt auf den Methodikseiten der Bücher angeboten. Diese sollen die Schüler/innen auf Wort-, Satz- und Textebene bei produktiven Handlungen zur Auswertung geographischer und besonders diskontinuierlicher Texte unterstützen. Der konkrete Unterschied zu Punkt 7 besteht darin, dass sich hier die sprachlichen Hilfen nur auf methodische Arbeitsweisen be- 
ziehen und nicht auf ein inhaltliches Problem im Rahmen der Themen auf den Doppelseiten.

9. Umfangreiche Methodikexkurse II - „How to work with... ": Ebenfalls im Kontext der Methodikseiten ist es in diesem Konzept das Ziel, Beschreibungen von Schritten zur Auswertung von geographischen Materialien als Informationsquelle vorzunehmen. Dies funktioniert umfangreich und auf verschiedenen Ebenen. Diese Ebenen sind: 1. Die Darstellung eines exemplarischen geographischen Materials oder einer Quelle (Karte, Diagramm etc.), um anhand eingeblendeter Wörter des Methodikund Fachwortschatzes den Aufbau und die Struktur der Materialien zu visualisieren, 2. die Bereitstellung von Informationstexten, welche Vorschläge für Analyse- und Beschreibungsschritte sowie Formulierungshilfen für Auswertungen geographischer Medien beinhalten und 3. Modelltexte, die konkrete Textvorgaben zu einer bestimmten sprachlichen Handlung vorschlagen, bspw. die Beschreibung einer thematischen Karte zur Landnutzung in Kalifornien.

10. Communication Skills: Vor allem allgemeinsprachliche Hilfen werden in einem Exkurs anhand von Satzanfängen, Phrasen und ganzen Sätzen dargestellt. Da diese Hilfsangebote in der Gesamtheit strukturell Texte vorgeben, fördern sie ebenfalls Fähigkeiten, die die Textkohärenz sprachlicher Handlungen betreffen, z. B. wie eine Diskussion oder eine Präsentation aufgebaut sein sollte.

11. Glossar/Wortlisten - Fachwortschatzkonzept, das nicht im Doppelseitenkontext stattfindet: Mit rezeptiven Vermittlungsstrategien in Form von Glossaren, Wort- und Vokabellisten werden in diesem Konzept Wortbedeutungen vermittelt. Der Vermittlungsumfang variiert zwischen reinen Ein-Wort-Übersetzungen und Beispielen zur Verwendung der Worte in einem Satz, bis hin zu Definitionen, die einen ganzen Abschnitt umfassen.

12. Signal Words / Operators: Hier wird anhand rezeptiver Informationstexte Transparenz über die Anforderungen von Sprachhandlungen hergestellt, die durch Operatoren und Signalwörter in den Aufgabenstellungen eingefordert werden.

13. Visualisierung und Erklärung von Zahlen und Maßeinheiten: Aus einer Kombination von Visuals und Informationstexten wird hier durch rezeptive Vermittlung auf die korrekte Bezeichnung und Verwendung von Zahlen und Maßeinheiten hingewiesen.

14. Orientation Pages: Auf den Exkursseiten der ,orientation pages' geht es schwerpunktmäßig darum, wichtige Informationen anhand produktiver Anforderungen in den Kontext zu setzen und Inhalte aus vorangegangenen Seiten mit Methoden wie Mind-Mapping zu wiederholen.

15. Exercise Pages: Auf den Übungsseiten, die außerhalb des Kontextes der Doppelseiten stehen, finden schwerpunktmäßig sprachlich rezeptive und produktive Übungen wie Lückentexte oder die Wortschatzübung, Außenseiter ${ }^{2}$ statt.

Vergleicht man die vorgestellten sprachlichen Förderkonzepte in den analysierten bilingualen Geographieschulbüchern, so fällt auf, dass die kontextuelle und strukturelle Integration der Hilfen unterschiedlich stark ist. Des Weiteren beziehen sich die Hilfen auf unterschiedliche sprachliche Teilkompetenzen. Ebenfalls essentiell ist, auf welchen Ebenen (Wort-, Satz- oder Textebene) die sprachlichen Hilfen greifen. Auf die genannten Unterschiede wird in den folgenden Abschnitten eingegangen.

\subsection{Sprachliche Hilfen im Bezug zur Wort-, Satz- und Textebene}

Die Ergebnisse zeigen, dass die Autor/innen der bilingualen Schulbücher einen Schwerpunkt in der Förderung des (Fach-)Wortschatzes der Schüler/innen setzen. Diese Schwerpunktsetzung findet sich in allen Büchern, unabhängig vom Verlag und von der Jahrgangsstufe, für die sie konzipiert wurden. 67,3\% (Abb. 2) der analysierten sprachlichen Hilfen bezie-

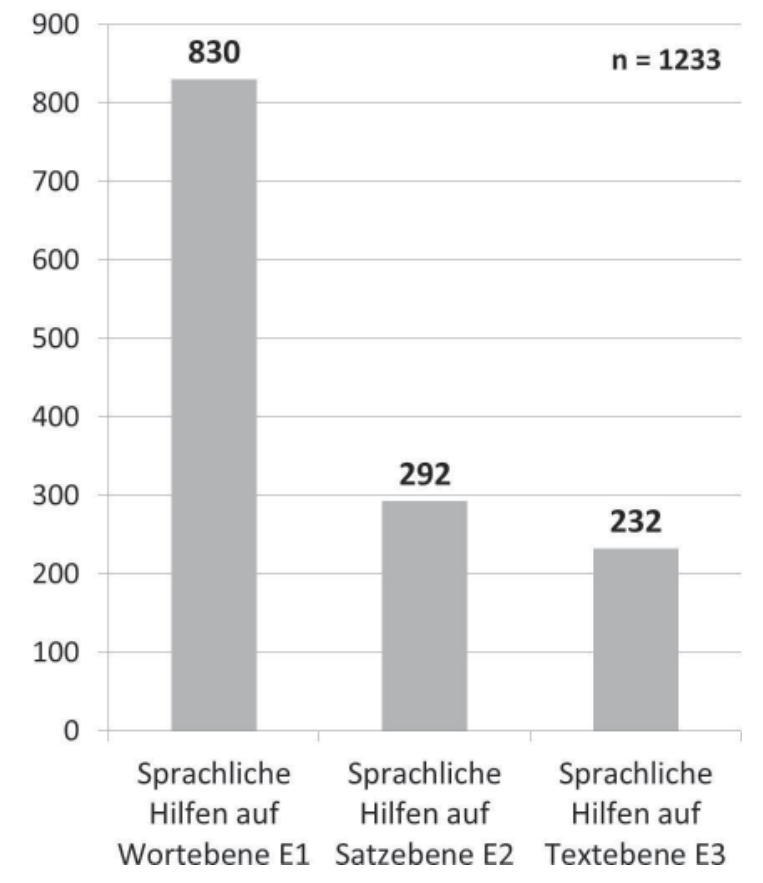

Abb. 2: Sprachliche Hilfen in Bezug zur Wort-, Satz-und Textebene (eigene Darstellung)

2 In dieser Übung muss aus einer Reihe von Begriffen ein Begriff identifiziert werden, der logisch nicht in die Reihe passt. 


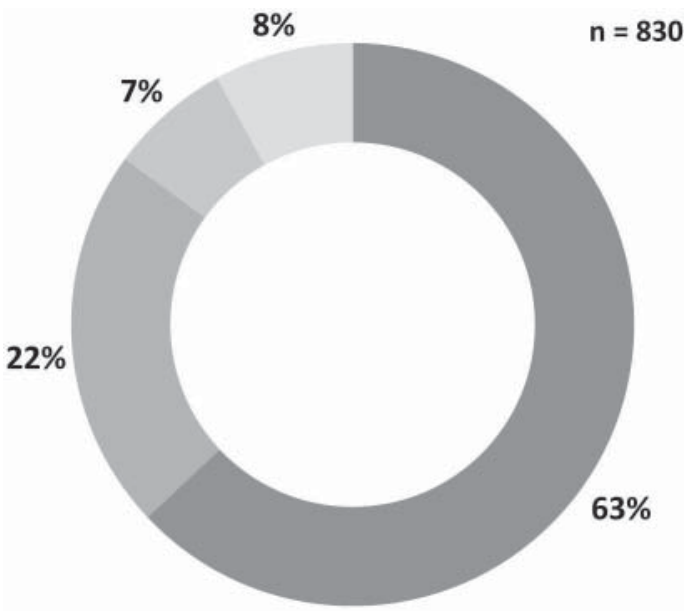

- Key Terms im Kontext

- Visuals/Layer im Kontext

Exkurs: Glossar/Wortlisten

Exkurs: Fachbegriffe in Methodikseiten

Abb. 3: Anteil der Sprachbilfen im Bereich Wortebene/Fachwortschatz (eigene Darstellung)

hen sich auf die Wortschatzarbeit (dies entspricht E1 in Abb. 1). Diese Förderung findet am häufigsten im Kontext einer Doppelseite statt. Hierbei wird zum Beispiel das Layerprinzip angewendet. Dies bedeutet, dass Begriffe direkt im Rahmen einer Abbildung eingeblendet, erklärt und visualisiert werden. Zudem werden Fachbegriffe auch in Abbildungen integriert oder über Fußnoten am Rand erklärt (siehe Punkte 5 und 8). Weiter zeigt Abb. 3, dass der Großteil der Förderkonzepte $(63 \%)$ auf Wortebene über die key terms (siehe Kapitel 4.1) im Bereich des Doppelseitenkontextes abgewickelt werden. Konzepte zur sprachlichen Förderung auf Satzebene sind in den untersuchten bilingualen Büchern ebenfalls vorhanden (dies entspricht E2 in Abb. 1). In der Regel werden helpful phrases angeboten. Diese sind von den Autor/ innen als sinnvoll erachtete Phrasen, Satzanfänge und Satzkonstruktionen zu verstehen, die den Schüler/innen bei Satz- und Textkonstruktionen helfen sollen. Die Schüler/innen müssen dann Sätze beenden, diese flexibel auf den Kontext beziehen, Wörter in Satzvorgaben einbetten und Phrasen übernehmen.

Die sprachlichen Hilfen, die die Textebene adressieren und Textkompetenz fördern, sind einerseits als helpful phrases zu verstehen, die ganz konkret einer Texthandlung zugeordnet werden können, was zum Beispiel durch die Überschrift der helpful phrases passiert, und / oder die in einem strukturellen Ablauf präsentiert werden. Damit ist gemeint, dass die Angebote der helpful phrases in einem textkohärenten Zusammenhang präsentiert werden, bspw. für Einleitungen, für Aufzählungen oder Schlussformulierungen. An- dererseits sind Hilfen auf Textebene zu verstehen, die konkret dabei helfen, Texte zu produzieren, interaktiv vorzutragen oder in eine andere Textform zu transferieren. Dies passiert über Modelltexte zu sprachlichen Handlungen oder über Hilfstexte, die Strukturierungen als Checkliste (siehe 4.1). Beispielsweise schlägt ein Modelltext folgende strukturelle und inhaltliche Formulierungen vor (hier das Beispiel zur Beschreibung einer thematischen Karte):

1. Einleitung: Quellenangabe, Seitenzahl, Titel und Maßstab

2. Lokalisierung: Gradnetz, flächenmäßige Eingrenzung des Gebiets, topographische Grenzen des Gebiets

3. Generelle Einleitung des Themas: Fokussierung auf dominierende Nutzungsformen

4. Spezifizierung des Themas: Ausschärfung der Nutzung in einzelnen Teilgebieten der Region

5. Infrastrukturelle Verbindung

6. Zusammenfassung: Darstellung der essentiellen Nutzungsformen (vgl. Westermann 2013: 193)

\subsection{Die Beziehung von Aufgabenstellungen und sprachlichen Hilfen}

Ferner zeigt sich, dass ein Großteil der Aufgabenstellungen in den Schulbüchern (95\%) keine Nutzung dieser Sprachhilfen einfordert, bzw. so auch nicht die Schüler/innen darauf hinweist, dass zur Behandlung der Aufgabe überhaupt sprachliche Hilfen zur Verfügung stehen. Es bleibt also hauptsächlich den Lehrer/ innen und den Schüler/innen überlassen, ob und wann sie diese idealtypischen Sätze und Phrasen einbinden.

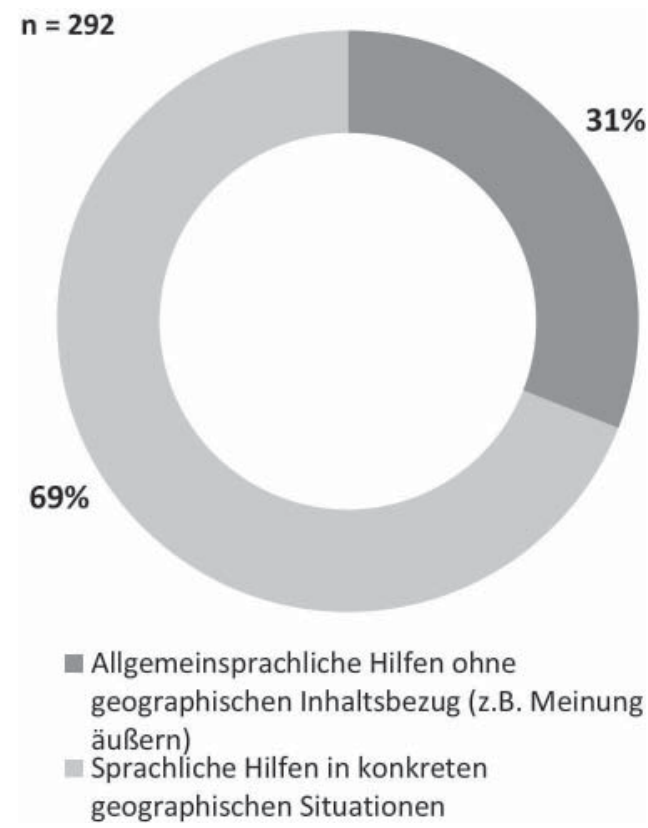

Abb. 4: Sprachförderkonzepte auf Satzebene: Helpful phrases in Bezug zu den Anforderungsbereichen der zugehörigen Aufgaben (eigene Darstellung) 

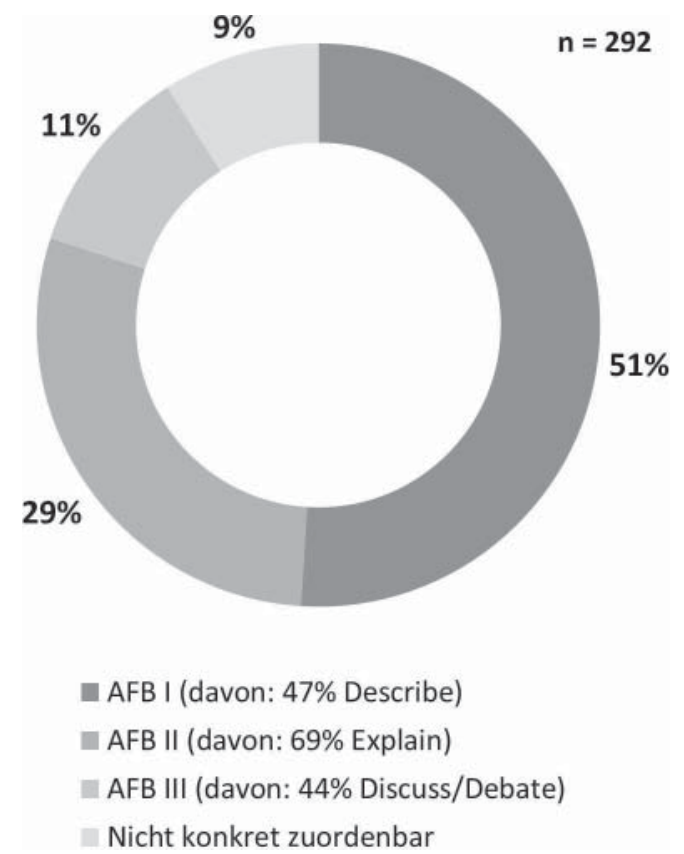

Abb. 5: Geographischer und allgemeinsprachlicher Bezug der Sprachhandlungen (eigene Darstellung)

Die angebotenen Blöcke von helpful phrases, die auf der Satzebene auftauchen, können nach den angestrebten Sprachhandlungen analysiert werden. Diese Zuteilung erfolgt zum einen durch den direkten Titel des Phrasenabschnitts oder über die zugehörige Aufgabe und die darin enthaltenen Signalwörter. Sprachhandlungen stehen, wie in 2.2 genannt, im Zusammenhang mit den Operatoren, die als Signalund Aufforderungswörter verstanden werden, welche die Handlung bestimmen. Dabei wird eben davon ausgegangen, dass die sprachlichen und inhaltlichen Anforderungen an die Schüler/innen mit den Anforderungsbereichen steigen. Wenn die Sätze z. B. mit "how to express your opinion" überschrieben sind, deutet dies darauf hin, dass sie auf das Ausdrücken der eigenen Meinung und die Formulierung von Beurteilungen vorbereiten sollen. Diese Anforderung kann dem AFB III nach den deutschen Bildungsstandards (DGfG 2014: 31) zugeordnet werden. Über die Hälfte der Hilfen auf Satzebene beziehen sich auf den AFB I. Dabei soll schwerpunktmäßig die Beschreibung geübt werden (siehe Abb. 4).

Weniger als ein Drittel der Sprachhilfen auf Satzebene bezieht sich auf den AFB II. Hier liegt der Schwerpunkt auf explain. Nur wenige der Sprachhilfen (11\%) sind für die Lösung von Aufgaben im höchsten AFB gedacht. Weiter wurde untersucht, ob es sich um allgemeinsprachliche Hilfen handelt oder um Hilfen, die konkret in einem geographischen Kontext positioniert sind (vgl. Abb. 4). Allgemeinsprachliche Hilfen sind losgelöst vom geographischen Kontext und behandeln sprachliche Handlungen, die vermehrt dem Sprachenunterricht entstammen. Geographiespezifische Hilfen zeichnen sich dadurch aus, dass verstärkt geographische Fachbegriffe integriert sind und geographiespezifische Zusammenhänge thematisiert werden. $69 \%$ der sprachlichen Hilfen sind in einen konkreten geographischen Inhalt mit Raumbeispiel eingebettet. $31 \%$ der Sprachhilfen wurden als allgemeinsprachlich identifiziert (siehe Abb. 4).

\subsection{Sprachliche Hilfen bezogen auf die sprachli- chen Dimensionen Produktion, Interaktion, Rezeption und Mediation}

Die Analyse zeigt, dass sich die sprachlichen Hilfen vor allem auf die Förderung rezeptiver und produktiver sprachlicher Anforderungen im Geographieunterricht beziehen (siehe Abb. 6).

In weniger als 5\% der analysierten Aufgaben und der dort eingesetzten Sprachhilfen kommt es zur Kopplung mit interaktiven Aufforderungen wie Peer-Review-Verfahren oder anderen kooperativen Elementen.

Knapp 5\% der Aufgaben sind überhaupt explizite Interaktionsaufgaben. Bei den auftauchenden Interaktionsaufgaben zeigt sich, dass in $51 \%$ der Aufgaben die Signalwörter discuss oder argue verwendet

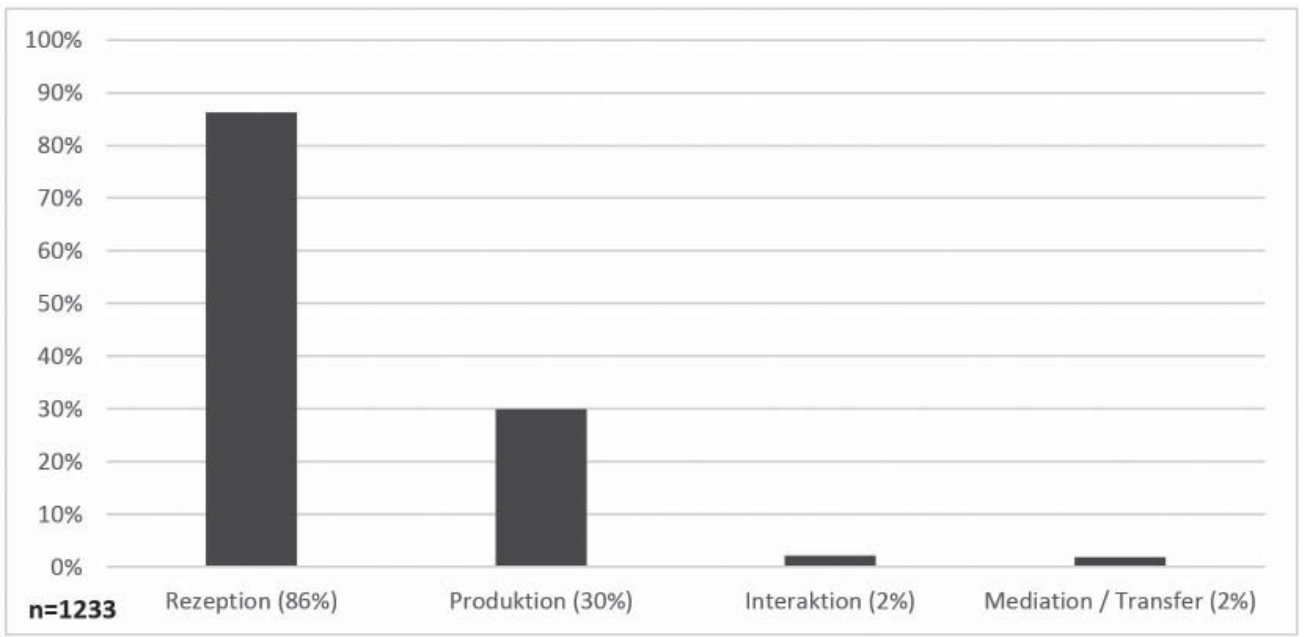

Abb. 6: Sprachliche Dimensionen, auf die sich die sprachlichen Hilfen beziehen (eigene Darstellung) 
werden. In $95 \%$ der Fälle geht es hier darum, dass die Schüler/innen argumentativ Fachinhalte erarbeiten und austauschen sollen. In 5\% der Fälle sollen sie in einer Partner- oder Gruppenarbeit angefertigtes oder erhaltenes Material hinsichtlich des Nutzens für die Beantwortung einer Problemfrage beurteilen. Somit kann hier eine Adressierung höherer Anforderungen nachgewiesen werden. In noch weniger Fällen wird im Rahmen einer Bereichsadressierung von Mediation und Transfer ein expliziter Bezug zu Konzepten von Alltagssprache und Fachsprache hergestellt.

Wenn im Bereich der Sprachmediation eine Aufgabe gestellt wird, geht es hauptsächlich um Textsortenwechsel (z. B. Umwandeln eines Textes in ein Fließdiagramm). Implizit nehmen die Schulbuchautorinnen und -autoren in wenigen Sprachhilfen Bezug zu Konzepten von Fachsprache und Alltagssprache. Dies geschieht beispielsweise wenn Schüler/innen dazu aufgefordert werden, einen fachsprachlichen Text aus der Sicht der betroffenen Akteur/innen zu formulieren oder einen Brief an ihre Großeltern zu schreiben.

\subsection{Qualitative Analyse der Methodikseiten}

Die Methodikseiten sind als Trainings- oder Förderelemente zu verstehen, mit denen die Schüler/innen Arbeitsweisen erlernen sollen, um geographische Inhalte aus Karten, Diagrammen oder Bildern zu erschließen bzw. um diese selber anfertigen zu können.

Interviews mit Lehrkräften, die Geographie bilingual unterrichten, haben ergeben, dass ein großer Teil gerade die Kombination von Verfahren in den Methodikseiten als essentiellen Baustein für die sprachliche Förderung einschätzt (vgl. Morawski \& Budke 2017a). Daher wurden die Methodikseiten genauer analysiert.

Die Methodikseiten liegen in der Regel nicht im Doppelseitenkontext, sondern am Anfang oder am Ende der Bücher. Durch gelegentliche Hinweise in relevanten Aufgabenstellungen auf den Doppelseiten werden Schüler/innen und Lehrer/innen auf die Verwendung dieser Seiten hingewiesen. Auf diesen wird dann strategisches Wissen zur geographischen Arbeitsweise mit dem Material explizit gemacht. Wie in Kapitel 4.1 erläutert, ist es in diesem Konzept das Ziel, die Arbeitsschritte im Rahmen der Auswertung von geographischen Materialien vorzunehmen. Dieses Ziel wird durchgesetzt, indem die Schüler/innen rezeptiv mit Texten über die Auswertungsschritte, mit dem Aufbau des Materials und mit sprachlichen Hilfen zur eigenständigen Produktion arbeiten sollen. Die Seiten beginnen in der Regel mit einer Darstellung des zu untersuchenden Materials. Beispielsweise wird eine thematische Karte abgebildet. Auf dieser werden in einer eingeblendeten Schicht der Wortebene fachinhaltliche Begriffe aus dem Fachwortschatz wie ,Breitengrad' oder ,Legende', aber auch allgemeinsprachliche Begriffe wie ,unterstrichen' oder, Gitter' und ,Linie' eingeführt. Auf diese Weise erkennen die Schüler/innen direkt in der Visualisierung des Materials, was unter dem jeweiligen Begriff zu verstehen und wie er zu verwenden ist. Diese Technik der Schichtung ist auf der Satzebene ebenfalls relevant. Wenn z. B. die Beschreibung eines Liniendiagramms geübt werden soll, kann eine Grafik genutzt werden, in welche allgemeinsprachliche und fachsprachliche Hilfen auf Wort- und Satzebene integriert sind.

Neben der visualisierenden Layertechnik werden den Schüler/innen auf den Methodikseiten Modelltexte angeboten, die zum Beispiel aus einer kompletten Beschreibung des jeweiligen Mediums bestehen können. Die Schüler/innen lernen auf diese Weise die Diskursfunktion ,Beschreiben' genauer kennen. Durch den Modelltext wird gezeigt, was unter einer geographischen Beschreibung zu verstehen ist. Weiter können sprachschwache Schüler/innen dem Modelltext u. a. hervorgehobene wichtige Begriffe und ganze Sätze entnehmen, die sowohl dem sprachlichen Fachwortschatz als auch dem allgemeinsprachlichen Wortschatz entstammen. Anzumerken ist hier, dass es sich bei den Modelltexten schwerpunktmäßig um Beispieltexte handelt, die Anforderungen im Bereich der basalen und kommunikativen Handlungen im AFB I wie ,beschreiben' oder ,herausarbeiten' umfassen. Die Herausforderung besteht für die Schüler/innen im Besonderen darin, sprachliche Elemente für den neuen Kontext zu übernehmen und dabei die sprachlichen und inhaltlichen Elemente zu streichen, die sich nur auf das Beispiel im Modelltext beziehen und nicht übertragbar sind.

In der Analyse konnte aufgezeigt werden, dass sich in allen untersuchten Lehrwerken die Methodikseiten hauptsächlich mit der Auswertung von thematischen Karten, Bildern, Balkendiagrammen und Klimadiagrammen befassen. Weiter sollen alle analysierten Methodikseiten rezeptive (z. B. Bilder oder Karten lesen) und produktive (eigenständige Textproduktion, eigene Beschreibung, Anfertigung eines Diagramms usw.) Kompetenzen fördern. Es sind dagegen wenige bis keine Konzepte zur Förderung der sprachlichen Interaktionskompetenzen der Schüler/innen vorzufinden, d.h. Aufgaben oder Verfahren, in denen die Schüler/innen die Möglichkeit bekommen zu lernende sprachliche Mittel mit einem Partner oder einer Partnerin einzuüben.

\subsection{Einsatz der deutschen Sprache in bilingualen Schulbüchern}

Die Schulbücher wurden im Bereich des angebotenen Materials, der Aufgabenstellungen, der Kon- 
zepte zur Fachwortschatzförderung und den Methodikseiten dahingehend untersucht, inwiefern die deutsche Sprache und zu welchem Zwecke sie in diesen Bereichen eingesetzt wird. Die Konzepte zum strategischen Einsatz der deutschen Sprache in den bilingualen Schulbüchern lassen sich nach zwei Zielsetzungen aufteilen (siehe Tab. 2). Unter dem Fokus funktionale Zweisprachigkeit ist zu verstehen, dass das Material in besonderer Weise den Wissenserwerb und damit verbundene Sprachhandlungen in beiden Sprachen (Deutsch und Englisch) ermöglicht. An dieser Stelle sind zur besonderen Unterscheidung der Zielsetzungen schwerpunktmäßig die adressierten Sprachhandlungen der analysierten Aufgabenstellungen zu nennen, da durch Aufgabenstellungen maßgeblich und zu großen Anteilen die sprachlichen Handlungen angeregt werden. Die Aufgabenstellungen, die in Büchern zu finden sind, die das Ziel der funktionalen Zweisprachigkeit verfolgen, ermöglichen in ca. einem Sechstel der Fälle, dass Aufgaben in beiden Sprachen bearbeitet werden sollen und/ oder können. Ferner bietet das Glossar in diesen Büchern ausführliche Erklärungen und Definitionen in beiden Sprachen an.

Dahingehend setzt das Material mit dem Fokus des englischen Fremdsprachenerwerbs den Schwerpunkt auf Sprachhandlungen, die exklusiv in der Zielsprache Englisch durchgeführt werden und vom Material und den Aufgaben aus, keine Möglichkeit anbieten, zwischen den Sprachen zu wechseln. Auf Wortebene zeigt sich ein gewisser Widerspruch bei der Fachwortvermittlung durch das Konzept keyterms, welches Schlüsselbegriffe im Kontext der Doppelseiten einführt. Die sonst einsprachigen Bücher mit dem Schwerpunkt Fremdsprachenerwerb greifen bei Schlüsselbegriffen auf eine deutsch-englische Übersetzung zurück und nutzen das Glossar einsprachig. Im anderen Fokus der Zweisprachigkeit hingegen werden die Schlüsselbegriffe einsprachig im Doppelseitenkontext eingeführt und das Glossar ist zweisprachig (siehe Tab. 2). Dies zeigt, dass besonders die Unterscheidungsformen im Glossar und in den Aufgabenstellungen die unterschiedlichen Zielsetzungen transparent machen.
Lediglich ein Verlag und ein Drittel der Bücher lässt die funktionale Zweisprachigkeit, beispielsweise durch die Auswahl der zu verwendenden Sprache, in einigen Aufgaben zu. Die Mehrzahl der Bücher verfolgt somit, den Ergebnissen der Analyse folgend, das Ziel des Fremdsprachenerwerbs.

\section{Diskussion der Stärken und Schwächen der analysierten bilingualen Schulbücher im Bereich sprachlicher Förderung}

$\mathrm{Zu}$ Beginn wurde dargelegt, dass die sprachlichen Hilfen in den bilingualen Geographielehrwerken mit der Idee untersucht wurden, aus ihnen fachsprachliche Förderverfahren für monolinguale, sprachbewusste Geographieschulbücher zu entwickeln. Daher ist es notwendig, die Stärken und Schwächen der Hilfskonzepte in den bilingualen Werken zu analysieren, um Stärken zu identifizieren und common errors zu vermeiden. An dieser Stelle werden zentrale Ergebnisse der Interviews mit bilingual unterrichtenden Lehrer/ innen (vgl. Morawski \& Budke 2017a) aufgegriffen, die zur Einschätzung und Problematisierung des in der Schulbuchanalyse erhobenen Ist-Zustands beitragen.

\subsection{Potenzielle Stärken}

Im Vergleich der untersuchten bilingualen Schulbücher zu Geographiebüchern auf Deutsch (siehe auch: Budke \& Kuckuck 2015; Budke et al. 2016) zeigen die bilingualen Schulbücher vielfältigere Ansätze zur Sprachförderung. Die Verknüpfung von sprachlichen und visuellen Informationen wird sehr viel stärker genutzt. Dabei wird u. a. die Layertechnik zur Visualisierung und Einblendung von Fachbegriffen verwendet. Ferner nutzen die bilingualen Bücher umfangreiche Ansätze zur Förderung des (Fach-) Wortschatzes und zur Schulung der Kompetenzen im Bereich von Produktion und Rezeption. Weiter zeigt sich eine Vielzahl von sprachlichen Hilfen insbesondere auf der Satzebene (E2), die sich sprachlich direkt in eine geographische Kontextsituation im-

Tab. 2: Konzepte des strategischen Einsatzes der deutschen Sprache in den bilingualen Schulbüchern

\begin{tabular}{|c|c|c|}
\hline & Fokus: Funktionale Zweisprachigkeit & Fokus: Englischer Fremdsprachenerwerb \\
\hline Vorkommen & - weniger als ein Drittel der Bücher & - mehr als zwei Drittel der Bücher \\
\hline Aufgaben & $\begin{array}{l}\text { - etwa ein Sechstel der Aufgaben sind wahl- } \\
\text { weise in Englisch oder Deutsch zu lösen }\end{array}$ & $\begin{array}{l}\text { - Aufgaben sind einsprachig auf Englisch zu } \\
\text { lösen }\end{array}$ \\
\hline Fachwörter & $\begin{array}{l}\text { - Key Terms in der Doppelseite bleiben ein- } \\
\text { sprachig } \\
\text { - Glossar mit Definition geographischer Fach- } \\
\text { begriffe zweisprachig }\end{array}$ & $\begin{array}{l}\text { - Zweisprachigkeit bei den keyterms in der } \\
\text { Doppelseite } \\
\text { - Glossar einsprachig in Englisch }\end{array}$ \\
\hline Methodikseiten & - Methodikteil komplett in Englisch & - Methodikteil komplett in Englisch \\
\hline
\end{tabular}


plementieren lassen. Diese Hilfen auf der Satzebene in bilingualen Büchern besitzen gegenüber den monolingualen deutschen Erdkundebüchern einen Vorteil. Dieser besteht darin, dass im Gegensatz zu der allgemeinsprachlichen und kontextergänzenden Verwendung sprachlicher Hilfen in vielen deutschen Büchern die Integration bestimmter Hilfen wie der helpful phrases in den bilingualen Büchern direkt im inhaltlichen Kontext und Raum stattfindet, wo diese auch verwendet werden sollen.

Die strukturierten Hilfen der Methodikexkurse erinnern deutlich an internationale Konzepte (vgl. Cruz 2012; Nutta et al. 2011; Cruz \& Thornton 2013), die ebenfalls einen Schwerpunkt auf strategische und methodische Hilfen setzen. Die Kombination aus verschiedenen Konzepten und auf verschiedenen Ebenen der Methodikexkurse zeigt ein hohes Potenzial und wird von den interviewten Lehrkräften als wertvolles Werkzeug zur sprachlichen Förderung benannt. Ebenfalls sind die Modelltexte auf der Ebene der Textkompetenz (E3) mit ihrem Potenzial als Hilfestellung und Orientierungsrahmen, als eine innovative Idee für die sprachliche Unterstützung im sprachbewussten Unterricht zu bewerten. Diese findet man in diesem Umfang eher wenig bis gar nicht in monolingualen Büchern (vgl. Budke et al. 2016).

Die Idee, die Schüler/innen entscheiden zu lassen, in welcher Sprache sie die Aufgaben bearbeiten wollen, ist ebenfalls innovativ. In ähnlicher Weise könnte die Muttersprache von Schüler/innen, die Deutsch als Zweitsprache lernen, in den Geographieunterricht, der vorrangig auf Deutsch stattfindet, eingebracht und als Entlastung zur Erklärung von Sachverhalten eingesetzt werden. Interviews mit bilingual unterrichtenden Lehrkräften (Morawski \& Budke 2017a) zeigen ähnliche Ansätze, da viele Lehrkräfte geographische Inhalte in beiden Sprachen (Deutsch und Englisch) im Rahmen der funktionalen Zweisprachigkeit vermitteln wollen.

\subsection{Analysierte Schwächen}

Es sollten auch die Schwächen des bilingualen Materials dargestellt werden, um diese für den sprachbewussten Unterricht möglichst umgehen zu können.

Viele der sprachlichen und methodischen Hilfen der bilingualen Schulbücher adressieren basale kommunikative Handlungen in niedrigeren Anforderungsbereichen. Hilfen, die höhere Anforderungen, auch im Bereich der high order thinking skills ansprechen, findet man dagegen selten. Dies erscheint problematisch, da die inhaltliche Verarbeitungstiefe häufig nicht der Komplexität der behandelten Themen entspricht und die Schüler/innen auch sprachlich nicht auf die Formulierung von anspruchsvollen Bewertun- gen und Argumentationen vorbereitet werden. Dies ist allerdings zur Teilnahme an Diskursen in demokratischen Gesellschaften unbedingt notwendig.

Die Ergebnisse ähneln bisherigen Analysen von Aufgabenstellungen in deutschsprachigen Geographieschulbüchern. Diese haben ebenfalls ergeben, dass sich nur wenige Argumentationsaufgaben in den Schulbüchern finden (vgl. Budke 2012). Auch bei der Kartenarbeit werden nur selten Aufgaben im AFB III gestellt (vgl. Budke et al. 2016). Die Lehrer/innen kritisieren die Schwierigkeit der Texte, die aus umständlichen Formulierungen resultiert und häufig an authentischen Quellentexten mangelt.

Trotz der Bemühungen, die sprachlichen Hilfen in einen geographischen Kontext einzubinden, sind viele Hilfen in den bilingualen Geographieschulbüchern allgemeinsprachlich und separiert von einem geographischen Problem gehalten. Hieraus folgt, dass die sprachlichen Hilfen nicht fachspezifisch genug sind und von den Schüler/innen daher womöglich nicht auf die behandelten geographischen Themen bezogen werden können. Allgemeinsprachliche, vom Kontext gelöste, sprachliche Hilfen bergen die Gefahr, dass der Fokus auf rein sprachliche Strukturen ohne geographischen Schwerpunkt gelenkt wird. Die sprachlichen Hilfen, insbesondere auf Satz- und Textebene müssen im geographischen Kontext stattfinden. Den Schüler/innen im sprachbewussten Geographieunterricht muss ersichtlich sein, auch im Rahmen von Scaffolding ${ }^{3}$ (vgl. Gibbons 2002), warum ihnen ein Hilfskonzept dabei hilft, den Inhalt professioneller formulieren zu können und inwieweit dadurch ihre Fähigkeit erweitert wird, im Rahmen von Fragestellungen und Diskursen zu kommunizieren. Nur auf diese Weise kann in heterogenen Klassen sprachbewusster Fachunterricht motivierend und inhaltlich gestaltet werden.

Ferner ist es auffällig, in welch geringem Maße sprachliche Interaktionsprozesse durch bspw. peer-correction, think-pair-share Verfahren gefördert werden. Die Bedeutung des kooperativen Lernens im Sinne positiver Interdependenz, auch für das Sprachenlernen, ist in der Literatur bereits lange dargestellt (vgl. Brüning \& Saum 2011; Bahr 2010) und müsste daher verstärkt bei der Schulbuchgestaltung berücksichtigt werden.

Die Modelltexte der Methodikseiten sind in ihrer Form sehr starr und geben den Schüler/innen wenig Raum zur Realisierung kreativer Lösungen. Folglich kann es diesen schwer fallen, das modellhafte Vorgehen flexibel auf die konkreten geographischen Prob-

3 Die als Metapher zu verstehende Begrifflichkeit Scaffolding (Baugerüst) meint ein Unterstützungssystem im Fachunterricht im Kontext von Zweitsprachenerwerb, das sprachliches und fachliches Lernen integriert. 
lemstellungen anzupassen. Von den interviewten bilingual unterrichtenden Lehrkräften werden zudem die hohe Schwierigkeit der Texte, die Umständlichkeit der Formulierungen und ein Mangel an authentischen Quellentexten kritisiert (vgl. Morawski \& Budke 2017a).

Ähnlich wie in bisherigen Untersuchungen von Methodikseiten zur Kartenarbeit in deutschen Geographieschulbüchern zeigt sich auch in bilingualen Erdkundeschulbüchern, dass Methodikseiten vorrangig Aufgaben in den basalen sprachlichen Anforderungsbereichen wie ,beschreiben' und ,nennen“" aufweisen. Somit werden die Fähigkeiten der Schüler/innen zur Entschlüsselung von Diskursen und zur reflexiven Kartenkompetenz (vgl. Gryl et al. 2010) deutlich weniger gefördert.

Im Gegensatz zur Produktion und Rezeption ist der Bereich der Sprachmediation in den Sprachhilfen deutlich unterrepräsentiert. Schüler/innen werden selten aufgefordert, bestimmte Texte in leichtere Texte umzuwandeln oder fachsprachliche Zusammenhänge in der Alltagssprache zu beschreiben. Wygotski (1979) und Rincke (2010) bemerken, dass Fachsprache nicht nur als weiterentwickelte Alltagssprache, wie Cummins (1991) in seiner Theorie auslegt, anzusehen ist, sondern selbst stückweise unabhängig weiterentwickelt werden kann. Beide Standpunkte betonen, dass die reflektierte Auseinandersetzung mit Alltagssprache im Bezug zu fachsprachlichen Komponenten Vorteile für das Verständnis der Lernenden bringt. Diese liegen zum einen im bewussten Erkennen sprachlicher Eigenschaften der beiden Register und darin, dass Schüler/innen auf dem Weg zur Professionalisierung fachsprachlicher Kompetenzen motiviert werden, da alternative Sprachanlässe geschaffen werden. Lässt man die Mediation zwischen Registern oder auch Textsorten außer Acht, übersieht man ein wichtiges Potenzial.

\section{Fazit}

Bilinguale sprachliche Förderkonzepte können einen Mehrwert für die Sprachförderung im sprachbewussten deutschsprachigen Geographieunterricht darstellen. Die Stärken der bilingualen Lehrwerke wie die strukturierten rezeptiven und produktiven Herangehensweisen in der Arbeit mit geographischen, besonders diskontinuierlichen Materialien, die im Kontext des Themas eingebundenen helpful phrases, der Differenzierungsgedanke zwischen allgemeinsprachlicher und fachspezifischer, fachsprachlicher Förderung sowie die Visualisierungsansätze von Sprache (Layer) wurden herausgestellt. Diese können in besonderem Maße auf deutschsprachige Geographieschulbücher übertragen werden. Somit können sie dazu beitragen, dass auch sprachlich schwächere Schüler/innen die komplexen Inhalte verstehen und sich am Unterricht verstärkt beteiligen können.

Die genannten Defizite im Bereich der sprachlichen Interaktion und Mediation könnten durch die schrittweise durchzuführende Implementation von Sprachfeedback und peer-correction Aufgaben ausgeglichen werden. Zudem kann man diese Aufgaben als kooperative Sprachförderung in der Interaktion zwischen Lehrer/innen und Schüler/innen sowie zwischen Schüler/innen untereinander auf die Instruktion für den Ablauf geographischer Arbeitsweisen anwenden. Zudem müssten Aufgaben in höheren Anforderungsbereichen stärker durch Sprachhilfen unterstützt werden, wodurch das geographische Lernen vertieft werden kann.

Die bilingualen Geographieschulbücher sollten besonders im Bereich der sprachlichen Interaktion und der sprachlichen Unterstützung anspruchsvoller Aufgaben weiterentwickelt werden. Zudem könnten aus den bilingualen Lehrwerken fachsprachliche Förderansätze auf die Entwicklung monolingualer Geographieschulbücher übertragen werden, um sprachliche Heterogenität und Multilingualität in den Klassen gewinnbringend zu nutzen. Einige Vorschläge zu Umsetzungen von sprachlicher Förderung im Geographieunterricht durch bilinguale und multilinguale Methoden und Materialien finden sich u. a. in Budke \& Kuckuck (2017). Auf den Grundpfeilern einer reflektierten Analyse bisheriger Schwächen und Stärken in der Sprachförderung bestehen dann hohe Erfolgschancen für die Entwicklung und nachhaltige Umsetzung eines sprachbewussten Geographieunterrichts.

\section{Literatur}

Bahr, M. (2010): Kooperatives Lernen im Geographieunterricht. In: Praxis Geographie 40/12. S. 4-5.

BAMF - Bundesamt für Migration und Flüchtlinge (2015): Aktuelle Zahlen zu Aysl. http://www.bamf.de/ DE/Infothek/Statistiken/Asylzahlen/AktuelleZahlen/ aktuelle-zahlen-asyl-node.html (6.6.2016).

Becker-Mrotzek, M., K. Schramm, E. Thürmann \& H. J. Vollmer (2013): Sprache im Fach - Einleitung. In: ebd. (Hrsg.): Sprache im Fach. Sprachlichkeit und fachliches Lernen, Berlin: Waxmann. S. 7-24.

Bourdieu, P. (2005): Was heißt sprechen? Zur Ökonomie des sprachlichen Tausches. Mit einer Einführung von John B. Thompson. Wien: New Academic Press.

Bourdieu, P. \& J. C. Passeron (1971): Die Illusion der Chancengleichheit. Untersuchungen zur Soziologie des Bildungswesens am Beispiel Frankreich. Stuttgart: Klett. 
Brüning, L. \& T. Saum (2011): Schüleraktivierendes Lehren und Kooperatives Lernen - ein Gesamtkonzept für guten Unterricht. http://vielfalt-lernen.zum.de/images/6/62/Basisartikel_KL_2011.pdf (08.08.2016).

Budke, A. (2011): Förderung von Argumentationskompetenzen in aktuellen Geographieschulbüchern. In: Matthes, E. \& C. Heinze (Hrsg.): Aufgaben im Schulbuch. Bad Heilbrunn: Klinkhardt. S. 253-264.

Budke, A. (2012): „Ich argumentiere, also verstehe ich.“ Über die Bedeutung von Kommunikation und Argumentation für den Geographieunterricht. In: ebd. (Hrsg.): Diercke Kommunikation und Argumentation. Braunschweig: Westermann. S. 5-18.

Budke, A. \& G. Weiss (2014): Sprachsensibler Geographieunterricht. In: Michalak, M. (Hrsg.): Sprache als Lernmedium in allen Fächern. Baltmannsweiler: SchneiderVerl. Hohengehren. S. 113-133.

Budke, A. \& M. Kuckuck (2015): Argumentieren mit Karten. In: Jahnke, H., A. Schlottmann, M. Mose \& A. Strüver (Hrsg.): Visuelle Geographien. Tagungsband 315. (In Druck).

Budke, A. \& M. Meyer (2015): Fachlich argumentieren lernen - Die Bedeutung der Argumentation in den unterschiedlichen Schulfächern. In: Budke, A., M. Kuckuck, M. Meyer, F. Schäbitz, K. Schlüter \& G. Weiss (Hrsg.): Fachlich argumentieren lernen. Didaktische Forschungen zur Argumentation in den Unterrichtsfächern. Münster: Waxmann. S. 9-28.

Budke, A., M. Michalak, M. Kuckuck \& B. Müller (2016): Diskursfähigkeit im Fach Geographie - Förderung von Kartenkompetenzen in Geographieschulbüchern. In: Menthe, J. (Hrsg.): GFD-Tagungsband. (In Druck).

Budke, A. \& M. Kuckuck (2017): Sprache im Geographieunterricht. Bilinguale und sprachsensible Materialien und Methoden. Waxmann. Münster.

CEFR - Council of Europe (2011): CEFR - Common European Framework of Reference for Languages. https:// www.coe.int/t/dg4/linguistic/source/framework_en.pdf (29.01.2016).

Cruz, B. C. (2012): Social Studies Teacher Education: Promoting and Developing Inclusive Perspectives. Talking Diversity with Teachers and Teacher Educators: Exercises and Critical Conversations across the Curriculum. New York: Teachers College Press.

Cruz, B. C. \& S. J. Thornton (2013): Teaching Social Studies to English Language Learners, 2nd edition. New York: Routledge Publishers.

Cummins, J. (1991): Conversational and academic language proficiency in bilingual contexts. In: AILA Review 8. S. 75-89.

DESI-Konsortium (2008): Unterricht und Kompetenzerwerb in Deutsch und Englisch. Weinheim: Beltz.

DGfG - Deutsche Gesellschaft für Geographie e.V. (2014): Bildungsstandards im Fach Geographie für den Mittleren Bildungsabschluss mit Aufgabenbeispielen. Bonn: Selbstverlag DGfG.

Feilke, H. (2012): Bildungssprachliche Kompetenzen fördern und entwickeln. In: Praxis Deutsch 233. S. 4-13.
Feilke, H. (2013): Bildungssprache und Schulsprache am Beispiel literal-argumentativer Kompetenzen. In: BeckerMrotzek, M., K. Schramm, E. Thürmann \& H. J. Vollmer (Hrsg.): Sprache im Fach. Sprachlichkeit und fachliches Lernen. Münster: Waxmann. S. 113-130.

Gibbons, P. (2002): Scaffolding Language, Scaffolding Learning. Teaching Second Language Learners in the Mainstream Classroom. Portsmouth, NH: Heinemann.

Golay, D. (2005): Das bilinguale Sachfach Geographie. Eine empirische Untersuchung zum sachfachlichen Lernzuwachs im bilingual deutsch-französischen Geographieunterricht in der Sekundarstufe I (mit unterrichtsmethodischen Empfehlungen und erprobten Materialien für die Praxis) (Dissertation). Nürnberg: Hochschulverband für Geografie und ihre Didaktik.

Gryl, I., M. Horn, K. Schweizer, D. Kanwischer \& T. Rhode-Jüchtern (2010): Reflexion und Metaperspektive als notwendige Komponenten der Kartenkompetenz. Geographie und ihre Didaktik 38/3. S. 172-179.

Habermas, J. (1981): Umgangssprache, Bildungssprache, Wissenschaftssprache. In: ebd. (Hrsg.): Kleine politische Schriften I-IV. Frankfurt am Main: Suhrkamp. S. 340-363.

Hattie, J. (2003): Teachers make a difference. What is the research evidence? University of Auckland. New Zealand. https://cdn.auckland.ac.nz/assets/education/hattie/docs/teachers-make-a-difference-ACER-(2003).pdf (08.11.2016)

Hemmer I. \& M. Hemmer (2010): Interesse von Schüler/ innen an einzelnen Themen, Regionen und Arbeitsweisen des Geographieunterrichts - ein Vergleich zweier empirischer Studien aus den Jahren 1995 und 2005. In: ebd. (Hrsg.): Schülerinteresse an Themen, Regionen und Arbeitsweisen des Geographieunterrichts. Ergebnisse der empirischen Forschung und deren Konsequenzen für die Unterrichtspraxis. Geographiedidaktische Forschungen 46, Weingarten: Selbstverlag des Hochschulverbandes für Geographie und ihre Didaktik. S. 65-149.

Hemmer, M., I. Hemmer, A. Hüttermann \& M. Ullrich (2010): Kartenauswertungskompetenz. Theoretische Grundlagen und erste Überlegungen zu einem Kompetenzstrukturmodell. In: Journal of Geography Education 3/38. S. 158-171.

Hillers, E. (1984): Afrika in europäischer Sicht. Die Behandlung außereuropäischer Völker und Kulturen am Beispiel Afrikas in ausgewählten europäischen Erdkundelehrbüchern (=Studien zur Internationalen Schulbuchforschung 38). Braunschweig.

King, F. J., M. S. Goodson \& F. Rohani (2016): High Order Thinking Skills. Definition. Teaching Strategies. Assessment. www.cala.fsu.edu/files/higher_order_thinking_skills.pdf (11.10.2016).

Kniffka, G. \& B. Neuer (2008): Wo geht's hier nach Aldi? Fachsprachen lernen im kulturell heterogenen Klassenzimmer. In: Budke, A. (Hrsg.): Interkulturelles Lernen im Geographie-Unterricht. Potsdam: Universitätsverl.S. 121-135.

Kniffka, G. \& T. Roelcke (2016): Fachsprachen - Vermittlung im Unterricht. Paderborn: Ferdinand Schöningh. 
Kuckuck, M. (2014): Konflikte im Raum - Verständnis von gesellschaftlichen Diskursen durch Argumentation im Geographieunterricht. Geographiedidaktische Forschungen 54, Münster: Monsenstein und Vannerdat.

Marienfeld, W. (1976): Schulbuchanalyse und Schulbuchrevision: zur Methodenproblematik. In: Internationales Jahrbuch für Geschichts- und Geographieunterricht 17. S. 47-58.

Mayring, P. (2015): Qualitative Inhaltsanalyse. 12. Auflage. Weinheim: Beltz.

Meyer, C. (2003): Bilingualer Unterricht am AVG aus Schülersicht. In: Auguste-Viktoria-Gymnasium Trier (Hrsg.): 350 Jahre Bildung und Erziehung Auguste-Viktoria-Gymnasium. Trier: Festschrift. S. 212-222.

Michalak, M., V. Lemke \& M. Goeke (2015): Sprache im Fachunterricht: Eine Einführung in Deutsch als Zweitsprache und sprachbewussten Unterricht. Tübingen: Narr Francke Attempto.

Morawski, M. \& A. Budke (2017a). Learning With and By Language: Bilingual Teaching Strategies for the Monolingual Language-Aware Geography Classroom. The Geography Teacher 14, 2. S. 48-67.

Morawski, M. \& A. Budke (2017b). Language Awareness in Geography Education - An Analysis of the Potential of Bilingual Geography Education for Teaching Geography to Language Learners. European Journal of Geography 7/5. S. 61-84.

Morek, M. \&V. Heller, V. (2012): Bildungssprache - Kommunikative, epistemische, soziale und interaktive Aspekte ihres Gebrauchs. In: Zeitschrift für Angewandte Linguistik 57. S. 67-101.

MSW NRW - Ministerium für Schule und Weiterbildung des Landes Nordrhein-Westfalen (2007): Kernlehrplan für das Gymnasium - Sekundarstufe I (G8) in Nordrhein-Westfalen Erdkunde. http://www.schulentwicklung.nrw.de/lehrplaene/upload/lehrplaene_download/ gymnasium_g8/gym8_erdkunde.pdf (16.10.2016).

MSW NRW - Ministerium für Schule und Weiterbildung des Landes Nordrhein-Westfalen (2011a): Kernlehrplan für die Realschule in Nordrhein-Westfalen Erdkunde. https://www.schulentwicklung.nrw.de/lehrplaene/ upload/klp_SI/RS/EK/RS_Erdkunde_Endfassung.pdf (16.10.2016).

MSW NRW - Ministerium für Schule und Weiterbildung des Landes Nordrhein-Westfalen (2011b): Kernlehrplan für die Hauptschule in Nordrhein-Westfalen Gesellschaftslehre, Erdkunde, Geschichte/Politik. http://www. schulentwicklung.nrw.de/lehrplaene/upload/lehrplaene_download/hauptschule/GL_HS_KLP_Endfassung. pdf (16.10.2016).

Nutta, J. W., N. U. Bautista \& M. B. Butler (2011): Teaching science to English language learners. New York, NY: Routledge.

Passon, P. (2007): Evaluation von Fachlernen und Sprachlichkeit im Kontext bilingualer Bildung. Osnabrück: Universität Osnabrück.

Reich,K.(2014):InklusiveDidaktik-Bausteinefüreineinklusive Schule. Reihe: Inklusive Pädagogik. Weinheim: Beltz.

Rincke, K. (2010): Alltagssprache, Fachsprache und ihre besonderen Bedeutungen für das Lernen. In: Zeitschrift für Didaktik der Naturwissenschaften 16. S. 235-260.

Roelcke, T. (2010): Fachsprachen (3. Ed.). Berlin: Erich Schmidt.

Salinas, C., M. Franquiz \& M. Reidel (2008): Geography approaches for second language learners: Highlighting content and practice. In: The Social Studies 99/2. S. 71-76.

Schendzielorz, C. (2011): Anerkennung im Sprechen. Eine theoretische und empirische Analyse der sozialen Dimension des Sprechens. http://www.bibb.de/dokumente/pdf/ Mag_Schendzielorz.pdf (03.08.2016).

Schmölzer-Eibinger, S. (2013): Sprache als Medium des Lernens im Fach. In: Becker-Mrotzek, M., K. Schramm, E. Thürmann\& H.J. Vollmer (Hrsg.): Sprache im Fach. Sprachlichkeit und fachliches Lernen. Berlin: Waxmann. S. $25-40$.

Schreier, M. (2012): Qualitative Content Analysis in Practice. Los Angeles, London: Sage Publications.

Tajmel, T. (2012): Von der Alltags- zur Bildungssprache. Ausbildungsmodul „Durchgängige Sprachbildung“. http://www.schulentwicklung.nrw.de/materialdatenbank/nutzersicht/getFile.php?id=5182 (16.10.2016).

United Nations (2006): Final report of the Ad Hoc Committee on a Comprehensive and Integral International Convention on the Protection and Promotion of the Rights and Dignity of Persons with Disabilities. http:// www.un.org/esa/socdev/enable/rights/ahcfinalrepe.htm (14.10.2016).

Westermann Verlag (2013): Diercke Basic 1. Braunschweig: Westermann.

Wiegand, P. (2006): Learning and teaching with maps. London: Routledge.

Wygotski, L. S. (1979): Denken und Sprechen. Frankfurt am Main: Fischer. 